\title{
Acetylcholinesterase activity as a neurotoxicity marker within the context of experimentally- simulated hyperprolinaemia: An in vitro approach
}

\author{
Konstantinos \\ Kalafatakis ${ }^{1,2}$, \\ Vasiliki Gkanti ${ }^{1,3}$, \\ Connie A. Mackenzie- \\ Gray Scott ${ }^{3}$, \\ Apostolos Zarros ${ }^{1,3}$, \\ George S. Baillie ${ }^{3}$, \\ Stylianos Tsakiris ${ }^{1}$
}

\begin{abstract}
${ }^{1}$ Laboratory of Physiology, Medical School, National and Kapodistrian University of Athens, Athens, Greece, ${ }^{2}$ Henry Wellcome Laboratories for Integrative Neuroscience and Endocrinology, School of Clinical Sciences, Faculty of Medicine and Dentistry, University of Bristol, Bristol, UK, ${ }^{3}$ Gardiner Laboratory, Institute of Cardiovascular and Medical Sciences, College of Medical, Veterinary and Life Sciences, University of Glasgow, Glasgow, UK
\end{abstract}

Address for correspondence:

Dr. Stylianos Tsakiris, Laboratory of Physiology, Medical School, National and Kapodistrian University of Athens, 75 Mikras Asias Street, Goudi, GR - 11527 Athens, Greece.

E-mail: stsakir@med.uoa.gr, stsakir@gmail.com

\begin{abstract}
Hyperprolinaemia is characterized by increased tissue accumulation of proline (Pro) and is known to exert serious cognitive and/or neuropsychiatric symptomatology as a direct result of Pro accumulation in the brain. The aim of this study was to explore a putative link between experimentally-simulated hyperprolinaemia and the activity of acetylcholinesterase (AChE); a crucial neurotoxicity marker. In vitro experiments were undertaken on purified eel-derived AChE, as well as on adult mouse brain homogenates, in order to examine the effect of a spectrum of Pro concentrations $(3,30,500$, and $1000 \mu \mathrm{M})$ on this marker. Our data showed that although Pro exerted a significant inhibitory effect on pure AChE activity, mouse brain-derived membrane-bound AChE activity was found either unaltered or significantly increased following incubation with Pro. The use of AChE activity as a neurotoxicity marker within the context of experimentally-simulated hyperprolinaemia should be considered with caution and in parallel with a number of other experimental parameters.
\end{abstract}

Key words: Acetylcholinesterase, hyperprolinaemia, neurotoxicity, proline

The term "hyperprolinaemia" describes two distinct inherited metabolic disorders resulting in increased tissue accumulation of proline (Pro): Hyperprolinaemia type I and II. ${ }^{[1]}$ The first occurs due to Pro oxidase deficiency [Figure 1a], while the second is caused by the functional inadequacy of $\Delta^{1}$-pyrroline-5-carboxylic acid dehydrogenase [Figure 1a]; both disorders are associated with elevated Pro levels in the central nervous system (CNS), and in some cases can lead to the manifestation of epileptic, serious cognitive (e.g., mental retardation), and/or other neuropsychiatric symptomatology. ${ }^{[1,2]}$ However, the neurochemical mechanisms underlying these symptoms are poorly understood, while, to date, the experimental efforts of reliably simulating the CNS of hyperprolinaemic states have only resulted in certainty of the role of oxidative stress in its etiopathogenesis. ${ }^{[2]}$

In recent years, a number of studies have focused on the in vivo and in vitro effects of Pro on rat CNS acetylcholinesterase
(AChE) activity. ${ }^{[3-5]}$ Apart from its classical role in hydrolyzing acetylcholine, AChE is an extensively studied enzyme of paramount importance for synaptic integrity, neurite outgrowth, neurodevelopment, and apoptosis. Delwing et al. ${ }^{[3]}$ injected 12 -day-old Wistar rats with a single subcutaneous injection of Pro ( $12.8 \mu \mathrm{mol} / \mathrm{g}$ of body weight $)$ and subsequently isolated their cerebral cortices $1 \mathrm{~h}$ after

This is an open access article distributed under the terms of the Creative Commons Attribution-NonCommercial-ShareAlike 3.0 License, which allows others to remix, tweak, and build upon the work non-commercially, as long as the author is credited and the new creations are licensed under the identical terms.

For reprints contact: reprints@medknow.com

How to cite this article: Kalafatakis K, Gkanti V, Mackenzie-Gray Scott CA, Zarros A, Baillie GS, Tsakiris S. Acetylcholinesterase activity as a neurotoxicity marker within the context of experimentally-simulated hyperprolinaemia: An in vitro approach. J Nat Sc Biol Med 2015;6:S98-101 


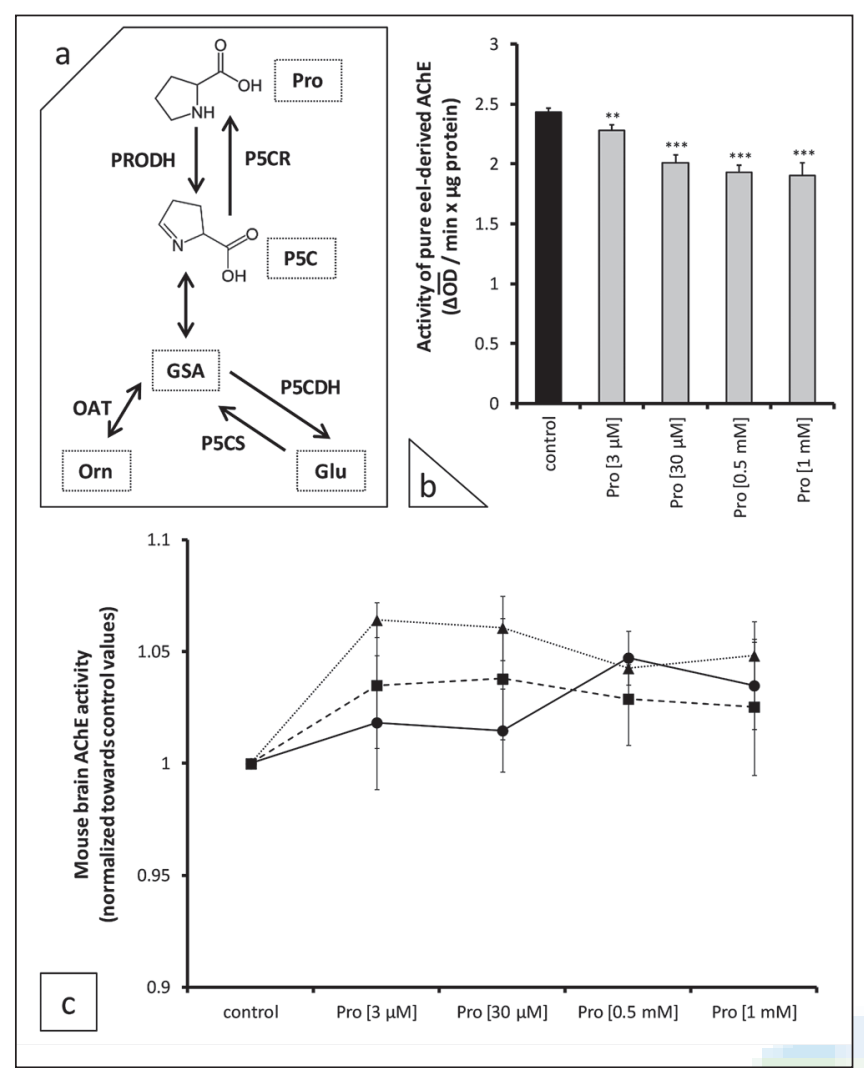

Figure 1: (a) Overview of the major metabolic pathway of proline (Pro); (b) Effects of different concentrations of Pro on eel-derived pure acetylcholinesterase (AChE) activity, in vitro; (c) Effects of different concentrations of Pro on the AChE activity of adult mouse brain homogenates, following a $30 \mathrm{~min}(\bullet-\bullet), 1 \mathrm{~h}(\mathbf{\square - - -})$ and $3 \mathrm{~h}(\boldsymbol{\Delta} \ldots \mathbf{\Delta})$ in vitro incubation. Notes: (a) Glu: glutamic acid, GSA: glutamic- $\gamma$-demialdehyde, OAT: ornithine aminotransferase, Orn: ornithine, P5C: $\Delta^{1}$-pyrroline-5-carboxylic acid, P5CDH: P5C dehydrogenase, P5CR: P5C reductase, P5CS: P5C synthase, PRODH: proline dehydrogenase (also known as proline oxidase). (b) The commercially-available (Sigma; AChE from Electrophorus electricus) pure enzyme was incubated at a concentration of $0.120 \mu \mathrm{g} / \mathrm{mL}$ in a reaction mixture containing $50 \mathrm{mM}$ Tris- $\mathrm{HCl}$, $\mathrm{pH} 8.0$ and $240 \mathrm{mM}$ sucrose in the presence of $120 \mathrm{mM} \mathrm{NaCl}$, according to the method of Ellman et al., ${ }^{[7]}$ as previously described in detail. ${ }^{[8]}$ The incubation of the pure enzyme with Pro (at various concentrations) lasted for $1 \mathrm{~h}\left(\right.$ at $\left.37^{\circ} \mathrm{C}\right)$; then, 5,5'-dithionitrobenzoic acid and acetylthiocholine iodide (substrate) were added to the reaction mixture (to a final concentration of 0.125 and $0.5 \mathrm{mM}$, respectively), and the occurring enzymatic reaction was followed by the changes in the absorbance $(\Delta \overline{\mathbf{O D}})$ at $412 \mathrm{~nm}$. Values indicate the mean \pm standard deviation of four measurements $(\mathbf{n}=4)$. ${ }^{* *} \mathbf{P}<0.01$; ${ }^{* * *} \mathbf{P}<0.001$. (c) Whole brains were obtained from four adult female C57BL/6 mice, were weighted, and then homogenized and centrifuged as previously described. ${ }^{[8]}$ The samples' protein concentrations were determined according to the method of Lowry et al. ${ }^{[9]}$ while their AChE activities after a 30 min, $1 \mathrm{~h}$ and $3 \mathrm{~h}$ incubation with Pro (at various concentrations; at $37^{\circ} \mathrm{C}$ ) were measured spectrophotometrically as previously described. ${ }^{[7,8]}$ Values indicate the normalized (toward the respective control) mean \pm standard error mean of four measurements $(\mathbf{n}=4)$. Murine brain homogenates exposed to any Pro concentration after a $3 \mathrm{~h}$ incubation demonstrates a significantly increased $(\mathbf{P}<0.05)$ AChE activity (as compared to respective control), in contrast to all other conditions (30 $\mathrm{min}, 1 \mathrm{~h}$ ) that did not significantly differ when compared to their respective control at any Pro concentration. the injection; through this approach, the rats were reported to have achieved plasma Pro levels of 1-2 mM (similar to those found in type II hyperprolinaemic patients) and CNS Pro levels of $0.25-0.55 \mathrm{mM}$. This acute administration of Pro resulted in a significant inhibition of rat cerebral AChE activity ( $-25 \%$ as compared to controls) and was prevented by pretreatment with established antioxidants (vitamin C or E). ${ }^{[3]} \mathrm{A}$ few years later, Delwing et al. ${ }^{[4]}$ repeated the same experiment on 29-day-old Wistar rats receiving a subcutaneous injection of Pro at a higher concentration $(18.2 \mu \mathrm{mol} / \mathrm{g}$ of body weight), and confirmed the significant inhibition of cerebral AChE activity due to Pro $(-32 \%$ as compared to that of controls). However, the undertaking of a more extended treatment scheme (where Pro was administered subcutaneously twice a day at $10 \mathrm{~h}$ intervals from day 6 to day 28 of age, at increasing doses ranging from 12.8 to $18.2 \mu \mathrm{mol} / \mathrm{g}$ of body weight) has failed to exert any significant effect on the rat cerebral AChE activity, despite the fact that the rats achieved (again) plasma Pro levels similar to those of hyperprolinaemic type II patients (1-2 mM). ${ }^{[4]}$ These findings formed the basis of a more recent study on male Wistar rats by Ferreira et al..$^{[5]}$ that used the same increasing dose Pro administration scheme but (i) either allowed for a washout period prior to the sacrifice of the rats on $60^{\text {th }}$ day of age or (ii) continued to administer Pro at the dose of $18.2 \mu \mathrm{mol} / \mathrm{g}$ of body weight from day 29 to day 60 of age (where sacrifice also occurred). Both treatment schemes failed to produce any significant changes with regards to AChE activity in the cerebral cortex of Protreated rats (as compared to saline-treated controls), but led to a significant increase of hippocampal AChE activity in hyperprolinaemic rats (as compared to controls). ${ }^{[5]}$

The effect of Pro administration on AChE activity has also been the subject of the recent study by Savio et al. ${ }^{[6]}$ on zebrafish (Danio rerio). Exposure at two Pro concentrations (1.5 and $3 \mathrm{mM}$ for $1 \mathrm{~h}$ or 1 -week) revealed that zebrafish brain AChE activity can only be significantly altered following the long-term (1-week) exposure to Pro ( $+34 \%$ and $+39 \%$ after exposure to 1.5 and $3 \mathrm{mM}$ Pro, respectively, as compared to controls). ${ }^{[6]}$ Interestingly, Savio et al..$^{[6]}$ also found that these increases in AChE activity were accompanied by significantly depressed expression of the ache gene, as demonstrated by quantitative real-time reverse transcription polymerase chain reaction assays; a finding suggesting that posttranslational events are likely to contribute to the Pro-induced effects on brain AChE activity.

Unfortunately, to date, the undertaking of in vitro experiments has not been of particular use toward the clarification of hyperprolinaemia-induced effects on brain AChE activity. ${ }^{[3,6]}$ The incubation of 12-day-old rat cerebral cortices' homogenates at $37^{\circ} \mathrm{C}$ for $1 \mathrm{~h}$ with various concentrations of Pro $(3,30,500$, and $1000 \mu \mathrm{M})$ 
demonstrates a concentration-dependent inhibition of AChE that was significant after exposure to 0.5 and $1 \mathrm{mM}$ Pro and reversible by vitamin E co-exposure. ${ }^{[3]}$ On the other hand, the in vitro effect of the same Pro concentration spectrum on zebrafish brain homogenates (tested at $25^{\circ} \mathrm{C}$ ) did not affect AChE activity at all. ${ }^{[6]}$

We herein present our findings with regards to the in vitro effect of exactly the same spectrum of Pro concentrations on pure eel-derived AChE activity [Figure 1b] as well as on adult female mouse (C57BL/6) brain homogenates' AChE activity following a $30 \mathrm{~min}, 1 \mathrm{~h}$ and $3 \mathrm{~h}$ incubation period [Figure 1c]. The determination of AChE activity was performed according to the method of Ellman et al. [7] as previously described in detail, ${ }^{[8]}$ while brain samples' protein determination was performed, according to the method of Lowry et al..$^{[9]}$ Obtained data were analyzed using one-way ANOVA followed by Bonferroni correction (where applicable), performed by SPSS (16.0) for Windows Software. Our data show: (i) that Pro provokes a direct, statistically significant inhibition of AChE activity at all tested concentrations [Figure 1b] and (ii) that adult murine brain homogenates exposed to any Pro concentration after a 3 h incubation present with a significant increase of AChE activity (as compared to controls), in contrast to shorter periods of incubation (30 min and $1 \mathrm{~h}$ ) where this increase is insignificant [Figure 1c].

These findings indicate that although Pro exerts a significant (concentration-dependent) inhibitory effect on pure AChE activity, brain-derived membrane-bound $\mathrm{AChE}$ activity is either unaltered or significantly increased following an incubation with Pro; an effect that introduces the possibility of Pro-induced modification of the lipid(s) $\mathrm{AChE}$ interactions as a regulator of $\mathrm{AChE}$ activity as in the case of other experimentally-simulated encephalopathies at the in vitro level. ${ }^{[8]}$ Considering the fact that Pro has also been found to provoke lipid peroxidation (as determined by an increase of thiobarbituric acid reactive species levels in the Pro-treated rat brain homogenates) following both acute $^{[10]}$ and long-term ${ }^{[11]}$ in vivo exposure of very young rats to the amino acid, the role of oxidative stress as an inhibitor of $\mathrm{AChE}^{[12]}$ proves to potentially be a major contributor to the reported in vivo acute Pro-induced AChE inhibition. ${ }^{[3,4]}$ However, oxidative stress is probably irrelevant with regards to the increase in membrane-bound AChE activity following a long-term in vivo exposure to $\mathrm{Pro}^{[5]}$ or an in vitro simulation of hyperprolinaemia as attempted on adult brain tissue by Savio et al. ${ }^{[6]}$ and ourselves [Figure 1c].

The current brief report summarizes the findings of an in vitro approach to the study of AChE activity as a neurotoxicity marker within the context of experimentally- simulated hyperprolinaemia, and has not reproduced any of the in vivo approaches (either acute or chronic) already reported in the literature. We believe that AChE activity should be considered as an in vivo neurotoxicity marker based on: (i) the extent of the treatment/exposure to Pro, (ii) the age/maturity of the laboratory animals' CNS at the sacrifice time point (as hyperprolinaemia seems to exert an age-dependent neurotoxicity), (iii) the extent of the undergoing lipid peroxidation and metabolic activity in hyperprolinaemic CNS tissues (parameters that must be co-examined in order to clarify the nature of the observed neurochemical changes), as well as (iv) a closer study on the effects of experimentally-induced hyperprolinaemia on sodium-potassium adenosine triphosphatase $\mathrm{Na}^{+}$, $\mathrm{K}^{+}$-ATPase) activity ${ }^{[1,13]}$ and/or other markers of excitotoxicity, ${ }^{[13]}$ that could well correlate with changes in AChE activity.

Finally, the examination of a wider CNS-region spectrum under both in vivo and in vitro conditions could shed more light on the nature and diversity of the effects of experimentally-simulated hyperprolinaemia on the activity of AChE; such an undertaking could provide us with more reliable tools toward the representation of this entity at an experimental level, which in turn may influence putative therapeutic strategies to counteract hyperprolinaemia.

\section{Acknowledgments}

The authors would like to acknowledge their appreciation to Dr. Christina Elliott (University of Glasgow) for her assistance in obtaining the necessary mouse brain tissues.

\section{Financial support and sponsorship}

Nil.

\section{Conflicts of interest}

There are no conflicts of interest.

\section{REFERENCES}

1. Mitsubuchi H, Nakamura K, Matsumoto S, Endo F. Inborn errors of proline metabolism. J Nutr 2008;138:2016-20.

2. Wyse AT, Netto CA. Behavioral and neurochemical effects of proline. Metab Brain Dis 2011;26:159-72.

3. Delwing D, Chiarani F, Delwing D, Bavaresco CS, Wannmacher $\mathrm{CM}$, Wajner $\mathrm{M}$, et al. Proline reduces acetylcholinesterase activity in cerebral cortex of rats. Metab Brain Dis 2003;18:79-86.

4. Delwing D, Chiarani F, Wannmacher CM, Wajner M, Wyse AT. Effect of hyperprolinaemia on acetylcholinesterase and butyrylcholinesterase activities in rat. Amino Acids 2005;28: 305-8.

5. Ferreira AG, Scherer EB, da Cunha MJ, Machado FR, Cunha AA, Graeff JS, et al. Physical exercise reverses cognitive impairment in rats subjected to experimental hyperprolinaemia. Neurochem Res 2011;36:2306-15.

6. Savio LE, Vuaden FC, Kist LW, Pereira TC, Rosemberg DB, Bogo MR, et al. Proline-induced changes in acetylcholinesterase activity and gene expression in zebrafish brain: Reversal by antipsychotic drugs. Neuroscience 2013;250:121-8. 
Kalafatakis, et al:: Acetylcholinesterase as a neurotoxicity marker of hyperprolinaemia

7. Ellman GL, Courtney KD, Andres V Jr, Featherstone RM. A new and rapid colorimetric determination of acetylcholinesterase activity. Biochem Pharmacol 1961;7:88-95.

8. Schulpis KH, Kalimeris K, Bakogiannis C, Tsakiris T, Tsakiris S. The effect of in vitro homocystinuria on the suckling rat hippocampal acetylcholinesterase. Metab Brain Dis 2006;21:21-8.

9. Lowry OH, Rosebrough NJ, Farr AL, Randall RJ. Protein measurement with the Folin phenol reagent. J Biol Chem 1951;193:265-75.

10. Ferreira AG, Lima DD, Delwing D, Mackedanz V, Tagliari B, Kolling J, et al. Proline impairs energy metabolism in cerebral cortex of young rats. Metab Brain Dis 2010;25:161-8.

11. Ferreira AG, Stefanello FM, Cunha AA, da Cunha MJ, Pereira TC, Bonan $\mathrm{CD}$, et al. Role of antioxidants on $\mathrm{Na}^{+}, \mathrm{K}^{+}$-ATPase activity and gene expression in cerebral cortex of hyperprolinaemic rats. Metab Brain Dis 2011;26:141-7.

12. Carageorgiou H, Tzotzes V, Pantos C, Mourouzis C, Zarros A, Tsakiris S. In vivo and in vitro effects of cadmium on adult rat brain total antioxidant status, acetylcholinesterase, $\left(\mathrm{Na}^{+}, \mathrm{K}^{+}\right)$-ATPase and $\mathrm{Mg}^{2+}$-ATPase activities: Protection by L-cysteine. Basic Clin Pharmacol Toxicol 2004;94:112-8.

13. Ferreira AG, da Cunha AA, Scherer EB, Machado FR, da Cunha MJ, Braga A, et al. Evidence that hyperprolinaemia alters glutamatergic homeostasis in rat brain: Neuroprotector effect of guanosine. Neurochem Res 2012;37:205-13.

\begin{tabular}{|l|l|}
\hline \multicolumn{2}{|c|}{ Access this article online } \\
\hline Quick Response Code: & Website: \\
\hline & www.jnsbm.org \\
\cline { 2 - 3 } & \\
\hline
\end{tabular}

